\title{
Access to Breast Cancer Treatment Services in Mombasa County, Kenya: A Quality of Care Analysis of Patient and Survivor Experiences
}

\author{
Sultane Sherman ${ }^{1,2}$, Vincent Okungu ${ }^{1, *}$ \\ ${ }^{1}$ Institute of Healthcare Management, Strathmore University, Nairobi, Kenya \\ ${ }^{2}$ Aga Khan University Hospital, Mombasa, Kenya \\ *Corresponding author: vokungu@strathmore.edu
}

\begin{abstract}
The increasing burden of cancer in Kenya has serious implications particularly for women. Understanding how women access breast-cancer treatment services is essential for mitigating its effects. The aim of this study was to examine access to breast cancer treatment services and implications on quality of care in Mombasa County, Kenya. Focus group discussions ( $\mathrm{N}=3$ ), in-depth interviews $(\mathrm{N}=7)$ and a questionnaire were used in data collection. Qualitative data were analyzed using preset themes of access. Other emergent themes were identified and analyzed. Quantitative data were analyzed in SPSS V.23 and tabulated as descriptive. Lack of equipment, distance to facilities, unavailability of specialized personnel, high cost of care and cultural stigma, were the main barriers of access to cancer services. These barriers impacted on quality of care in terms of timeliness, equity, effectiveness, patient-centeredness, safety and efficiency. Poor quality of care was exhibited in reports of wrong and late diagnosis, poor outcomes in surgery, severe burns, broken down equipment, among others. There were also social consequences of being diagnosed with breast cancer including loss of business and termination from employment. Concerted efforts including investments in health workers, equipment and awareness creation are required to support access to quality breast cancer care.
\end{abstract}

Keywords: Kenya, non-communicable diseases, breast cancer, access, quality of care

Cite This Article: Sultane Sherman, and Vincent Okungu, "Access to Breast Cancer Treatment Services in Mombasa County, Kenya: A Quality of Care Analysis of Patient and Survivor Experiences.” American Journal of Public Health Research, vol. 6, no. 4 (2018): 189-194. doi: 10.12691/ajphr-6-4-3.

\section{Introduction}

Kenya is one of 106 developing countries that lack access to medicine and experience a high burden of non-communicable diseases (NCDs) [1]. Of the NCDs, cancer could be the most devastating to households. Globally, cancer causes more deaths than HIV/AIDS, TB and malaria combined [2,3]. With over $70 \%$ of the global cancer burden in LMIC, The Economist [4] in a viewpoint publication, described cancer as worse than HIV/AIDS in the developing world and the burden is heaviest on the poor. In Kenya, the number of newly diagnosed cancer cases rose by $46.4 \%$ between the years 2012 (28,000 cases) [5] and $2015(41,000)$ [6]. It is estimated that daily cancer mortality rate in Kenya is the highest of the NCDs and registered a 6\% annual growth rate between 2010 and 2014. Currently, cancer is the leading cause of hospitalization and mortality at the Kenyatta National Hospital (KNH), Kenya's largest national referral facility [6]. The hospital's cancer registry indicates that $53 \%$ of all patients admitted in 2015 were due to cancer related morbidity. The rapid growth in cancer mortality rate suggests that cancer could be on course to be the leading cause of NCD-related deaths in Kenya. The deaths (about
$76 \%$ of all cases) are mainly caused by delays in treatment due to lack of personnel and equipment, and late detection of nearly $80 \%$ of reported cases. Nugent [7] suggests that because of late diagnosis, people developing NCDs such as cancer in many LMIC are more likely to die during their productive years ( $<60$ years) than after retirement. A recent study in Nairobi [8] shows that in terms of prevalence, prostate cancer (15.6\%) and breast cancer (23\%) were the most common types of cancers among men and women respectively. However, cancer poses a greater threat to Kenyan women than men. For the women, breast and cervical cancers affect 34 per 100,000 and 25 per 100,000 women, respectively. Among the men, esophageal, prostate cancer and Kaposi sarcoma have comparably lower incidence rates of 17.5, 15.2 and 9.2, per 100,000 men, respectively [9]. There is a positive link between female gender and NCDs $[10,11,12]$ and being female in Kenya increases the likelihood of NCD by 7.41\% [13].

Breast cancer is the most common type of cancer in Kenya. Few studies in Kenya have attempted to explore in detail whether breast-cancer patients have access to quality health services whenever they need them.

The objective of this study was to examine in detail access to breast cancer treatment services in Mombasa County with a focus on members of the Breast Cancer Survivors of Coast (BRECASCO). BRECASCO was 
formed to provide various forms of social support to its members.

\section{Methods}

A descriptive mixed methodology was used to generate detailed information about patient and survivor experiences in accessing breast cancer treatment services. The study took place in Mombasa County and involved members of the Breast Cancer Survivors of Coast (BRECASCO). The unit of analysis were individual members.

Qualitative data were collected using FGDs $(\mathrm{n}=3)$ and in-depth interviews ( $n=7)$. Data saturation [14] was achieved around the following topics: availability, affordability and acceptability of breast cancer services. Data were audio-recorded, transcribed analysed thematically by the researchers. Frequency counts of the number of issues and views expressed by type were undertaken.

For quantitative data, a formula $s=X^{2} N P(1-P) / d^{2}(N-$ 1) $+X^{2} P(1-P)[15]$ was used to calculate the sample size ( $\mathrm{s}=86$ out of 110 members). A standardized questionnaire was sent to a random sample of 86 consenting participants of which 72 questionnaires were filled and returned. Likert scale was used in the analysis. The data were cleaned, coded and analysed using SPSS v.23.0. The results were presented in summary tables. All data were interpreted from a quality of care lens.

\section{Limitations of the Study}

The study was conducted in an organized group of cancer survivors and patients and their level of access to services may not entirely reflect the realities of the county where other patients survive without support systems. There is a need for a larger study on access to breast cancer treatment services.

\section{Results}

Of the 72 study participants, 51\% were survivors and were no longer on treatment. Table 1 is a summary of sociodemographic characteristics of study participants.

From Table 1, the majority (70\%) of respondents are within the age range 36 - 55 years, suggesting that the burden of breast cancer is felt most among the most productive age population. About $43 \%$ have primary and secondary education and a similar number has post-secondary education. The single largest majority (about $46 \%$ ) is self-employed which indicates potential financial vulnerabilities because the majority of those outside formal employment in Kenya are uninsured. The fact that a significant number (43\%) were diagnosed with breast cancer five years ago or lower may suggest improved diagnostic skills, or an emerging breast cancer epidemic which is consistent with current epidemiological trends in the country. Among those that had survived cancer for 11 years and more, the majority (55\%) had secondary or post-secondary school education suggesting early diagnosis for those with better education.

Table 1. Socio-demographic characteristics of respondents

\begin{tabular}{|c|c|c|}
\hline \multicolumn{2}{|l|}{ Characteristic } & No. (\%) \\
\hline \multirow{4}{*}{ Marital Status } & Married & $36(49.6)$ \\
\hline & Divorced & $07 \quad(9.7)$ \\
\hline & Widowed & $13(18.1)$ \\
\hline & Single & $16(22.6)$ \\
\hline \multirow{3}{*}{ Age } & Below 35 years & $08(11.2)$ \\
\hline & $36-55$ years & $52(72.3)$ \\
\hline & Above 55 years & 12 (16.7) \\
\hline \multirow{3}{*}{ Number of children } & $1-2$ & $33(45.8)$ \\
\hline & $3-5$ & $31(43.1)$ \\
\hline & 6 and above & $11(11.1)$ \\
\hline \multirow{5}{*}{ Level of Education } & Never attended school & $07 \quad(9.7)$ \\
\hline & Primary school & $11(15.2)$ \\
\hline & Secondary school & $20(27.8)$ \\
\hline & Post-secondary & $31(43.1)$ \\
\hline & Other & $03 \quad(4.2)$ \\
\hline \multirow{5}{*}{ Employment Status } & Formally employed & $14(19.4)$ \\
\hline & Self-employed & $33(45.8)$ \\
\hline & Unemployed & $13(18.1)$ \\
\hline & Retired & 10 (13.9) \\
\hline & Other & $02 \quad(2.8)$ \\
\hline \multirow{4}{*}{ When diagnosed? } & $0-5$ years ago & 29 (43.3) \\
\hline & $6-10$ ago & 14 (20.9) \\
\hline & $11-15$ ago & $21(31.3)$ \\
\hline & Above 15 years & $3(4.5)$ \\
\hline
\end{tabular}

Most study participants had sought treatment from the public health sector because it is low cost. However, at least eight formally employed either sought treatment abroad $(n=3)$ or at local private facilities $(n=4)$. This is indicative of financial difficulties among those in non-formal employment. In terms of availability of breast cancer treatment services (Table 2), there are critical challenges particularly with access to timely and adequate essential services. About $76 \%$ of study participants either disagreed or strongly disagreed that they were served in the shortest time possible, suggesting long queues for the majority of patients.

From Table 2, up to $85 \%$ of respondents could not access necessary radiotherapy services and 53\% reported lack of adequate staffing to administer breast cancer treatment. Although 57\% agreed/strongly agreed that the facilities in which they sought treatment were nearby, a significant number still travelled long distances to seek treatment. Despite these challenges, the majority (96\%) received the drugs they needed even if they paid out-of-pocket.

Table 2. Availability of breast cancer treatment services

\begin{tabular}{|c|c|c|c|c|}
\hline \multicolumn{5}{|l|}{ Availability of breast cancer treatment services } \\
\hline Service availability & Strongly agreed (\%) & Agreed (\%) & Disagree (\%) & Strongly disagreed (\%) \\
\hline Services were timely (I did not have to wait for long) & 5 & 19 & 73 & 3 \\
\hline I did not have to travel long distance to get treatment & 17 & 40 & 36 & 7 \\
\hline The facility had all drugs to ensure proper treatment & 21 & 75 & 3 & 1 \\
\hline The full range of radiotherapy services is available & 5 & 10 & 56 & 29 \\
\hline There were adequate staff for my treatment & 5 & 43 & 43 & 9 \\
\hline
\end{tabular}


The qualitative component of the study confirmed the various challenges faced by breast cancer patients with regard to availability of services; e.g. long waiting periods running into months to see an oncologist. From the FGDs, lack of specialist services meant that some patients were treated by general practitioners (GPs), which compromised quality of care; e.g. one respondent reportedly suffered severe burns during her treatment cycle and had to nurse the wounds before resuming treatment. She said:

"I have never encountered such pain in my life; even child birth was not as painful. I couldn't wear clothes; I couldn't sleep on my back - it was a terrible time. I pray no woman would ever go through what I went through...."

Lack of specialist care is complicated by shortage of facilities with the capacity to treat breast cancer. Those relying on public services travel about $400 \mathrm{~km}$ to Nairobi e.g. for radiotherapy services. Persistent breakdown of radiotherapy machines complicated availability of these services and meant long spells of interrupted treatment cycles and costs to the patient.

Affordability of breast cancer services, as illustrated in Table 3, remains a key access barrier. Up to 94\% of the respondents agreed or strongly agreed that they needed financial support to access treatment. A large majority (81\%) had taken loans and $73 \%$ had sold at least an asset, to finance their treatment. About $54 \%$ potentially face catastrophic expenditures with treatment costs estimated at above $40 \%$ of total household expenditure.

The challenges on affordability of cancer services were emphasized by many respondents in the qualitative component of the study. The majority reported financial pressures on their households. At least three respondents delayed treatment for several months to raise enough money. The nock-on effect of breast cancer illness was vastly felt on employment and has serious potential for impoverishing households; e.g. one respondent had to shut down her small business because she used up the business capital for treatment. At least three FGD participants acknowledged that long absence from work led to their businesses incurring heavy losses and potential collapse. Two study participants reportedly lost their jobs either because of long absence from work or termination by an employer who got apprehensive about the potential longterm cost of treating cancer. Collapse of business or losing a job are huge challenges with respect to long-term care as expressed by a respondent:

"Losing my job felt like a second death sentence; how was I going to afford treatment costs when my only source of income was taken away from me?"
At least eighteen study participants reported loss of crucial time in their prognosis for lack of money. Many resorted to herbal remedies, borrowing and sale of assets to raise money for treatment. Some households cut on food expenditures. One participant said:

"At times I was forced to choose between buying food for my children and going for chemotherapy... It was a very painful decision."

For those who did not face financial challenges in their course of treatment, the role of both public and private insurance cover was apparent. The National Hospital Insurance Fund (NHIF) with its newly enhanced cover including surgery, chemotherapy and radiotherapy, was praised by patients who were members of the scheme. The NHIF coverage, even though has significant limits on cancer treatment, highlights the importance of public insurance, particularly for low-income populations.

On acceptability of services (Table 4), there was respect from health workers in terms of communication with patients and other forms of interaction. However, there was also some level of stigma given that at least 73\% of study participants agreed that it is against their culture to openly discuss breast cancer, which could limit communication with health workers.

From in-depth interviews and FGDs, participants questioned health worker skills and knowledge on breast cancer. Despite obvious lumps on their breasts, six study participants reported that they were given antibiotics for an undisclosed infection and spent several months treating symptoms before the right diagnosis was made. There were reports of poor outcomes with one respondent reporting being admitted three times before her mastectomy was performed and another developed serious wounds and she had to be taken back to theatre for a surgical toilet. One of the respondents had a poor mastectomy which resulted in re-growth of the cancer. She said:

"The government doctors do not know what breast cancer is and do not know how to treat it properly; so how can we be going to such people for treatment?"

Although $91 \%$ of study participants were positive about communication from health workers, at least 10 FGD participants reported poor communication from doctors. One respondent said:

"The doctor's approach was not good - the diagnosis of breast cancer felt like a death sentence...the communication was rather like, "go deal with it," which I found quite unfortunate coming from a government hospital."

Table 3. Affordability of breast cancer treatment services

\begin{tabular}{|c|c|c|c|c|}
\hline Service affordability & $\begin{array}{c}\text { Strongly } \\
\text { Agree (\%) }\end{array}$ & Agree (\%) & Disagree (\%) & $\begin{array}{c}\text { Strongly } \\
\text { Disagree (\%) }\end{array}$ \\
\hline I need financial help to access treatment & 65 & 29 & 5 & 1 \\
\hline I took a loan from the bank and /or friends/relatives to pay for treatment & 11 & 70 & 18 & 1 \\
\hline I have sold my assets to meet treatment costs & 8 & 65 & 24 & 3 \\
\hline Cost of treatment usually is more than $40 \%$ of household monthly expenditure & 19 & 35 & 31 & 15 \\
\hline
\end{tabular}

Table 4. Acceptability of breast cancer treatment services

\begin{tabular}{|c|c|c|c|c|}
\hline Acceptability of services & $\begin{array}{c}\text { Strongly } \\
\text { Agree (\%) }\end{array}$ & $\begin{array}{c}\text { Agree } \\
(\%)\end{array}$ & $\begin{array}{c}\text { Disagree } \\
(\%)\end{array}$ & $\begin{array}{c}\text { Strongly Disagree } \\
(\%)\end{array}$ \\
\hline Staff at the health facility were happy to serve me & 22 & 58 & 19 & 1 \\
\hline I was happy with the communication and feedback from health workers & 2 & 89 & 3 & 6 \\
\hline I believe that breast cancer is caused by spirits and bad omen & 5 & 14 & 76 & 5 \\
\hline In our culture, women cannot talk openly about breast cancer & 8 & 65 & 24 & 3 \\
\hline
\end{tabular}


Cultural perceptions about illness could mean important barriers to care seeking and acceptance of a prescribed treatment regimen. During the FDGs, some participants noted that the community perceived breast cancer as a curse, a taboo or witchcraft. This misconception contributed to collapse of immediate patient support such as family, friends and community, leading to lack of access to finances. This in turn leads to delays in treatment and poor health. A respondent said:

"My family, neighbors and relatives believed that cancer will always lead to death.... there was so much stigma, I was singled out by people and was deserted.... Being a breast cancer patient in this region can be very lonely."

A key informant indicated that it is because of such levels of stigma that they started the BRECASCO support group; first to provide social accommodation to breast cancer patients and survivors and also to raise awareness and address stigma in the larger community. Said a key informant: "This myth and perception about breast cancer makes women not to go for screening and testing." Literature shows that late diagnosis is the leading cause of cancer mortality worldwide.

\section{Discussion and Recommendations}

Timely access to quality health services is key to treatment outcomes. The findings of this study indicate poor access to quality breast cancer treatment services which leads to poor health outcomes. Service unavailability including long distance to facilities, lack of timely follow up, lack of specialists, lack of equipment, etc., have been documented in this study. For these factors, there is high potential for increased mortality from breast cancer in Mombasa County. There is already evidence suggesting that women in are more susceptible to NCDs than men $[10,11,12,13]$ and that the majority present late with the disease [11]. Joseph et. al., [16] reiterate that women who do not present for screening have a higher risk of disease progression. Besides, being treated by personnel not trained on oncology as well as interrupted treatment cycles raise questions around patient safety and treatment effectiveness. Not only did patients experience severe burns but also the risk of metastases leading to the spread of cancer to other organs. This study has linked patient safety and treatment effectiveness concerns to severe shortage of manpower with the right skill mix to diagnose and treat breast cancer. The shortage of skilled manpower including cancer specialists, potentially leads to misdiagnosis, poor surgical procedures which in turn result in more costs and poor health outcomes to the patient. Those diagnosed faced worsening illness as they waited on queues for months. Early detection and treatment ensure the most favorable clinical outcomes and enabling simpler and more cost-effective treatment. Some authors [17,18,19] have written on similar issues with regard to availability of health services. Kenya needs more oncologists trained and dispersed to referral hospitals in different counties. There is also a need to emphasize patient-centered care to make breaking news about breast cancer diagnosis bearable. Vardy and Tannock [20] emphasize the need for quality cancer care that is delivered with compassion.
Timeliness of interventions seems to be the most critical gap identified by the study both quantitatively and qualitatively. Other than being a consequence of shortage of skilled manpower, lack of timely interventions also emanated from cost of care, distance to facilities, not knowing where to seek care, severe shortage of critical equipment, long waiting times, local perceptions about illness and lack of awareness of symptoms.

Cost of services raised equity concerns as some patients could afford better quality care from private facilities while those opting for relatively cheaper public facilities faced longer delays to access treatment and adverse events. Sankaranarayanan et al.,[21] notes that cost of services results in wide variations in cancer survival between countries. Both direct and indirect out-of-pocket (OOP) costs of breast cancer treatment featured prominently in the findings. The social and economic consequences of OOP payments including depletion of business capital leading to business collapse, were noted. For households relying on OOP payments for cancer care job loss is a likely death sentence. The immediate and long-term consequence from OOP payments include serious financial shocks and impoverishment. McIntyre et. al., [22] give evidence of household impoverishment when faced with substantial medical expenses which are paid OOP. In resource poor settings, illness imposed high and regressive cost burdens on patients and their families[23]. Furthermore, the evident social and policy consequences of the disease including job losses from illegal termination and collapse of individual enterprises, are serious social issues that require immediate attention from policymakers and the society at large. To address affordability issues there should be concerted efforts especially by the national government to improve universal access to cancer treatment services.

Efficiency concerns were raised in the study where limited or broken down radiotherapy machines were noted. There is need to invest in acquisition and maintenance of these machines and make them available in clusters of counties to achieve cost-efficiency. Poor prognosis of patients is as a result of late diagnosis and poor treatment options [24,25] and can be linked to a lack of essential equipment and manpower, among other factors.

As this study demonstrates, lack of timely intervention can also be a consequence of cultural attitudes and lack of knowledge about breast cancer. Many respondents showed poor knowledge of breast cancer; i.e. that breast cancer is the result of evil spirits, certain foods and family planning implants. Such beliefs influence patients to seek unconventional 'healthcare' e.g. spiritual interventions, which further delay timely treatment. Many authors [26,27,28,29] have highlighted local illness perceptions which directly influence quality of care.

A critical element of culture that affects timeliness and effectiveness of care is stigma. Stigma, as noted in the findings, denied some patients their social support systems including income. Lack of such support and in particular, a lack of intimate confiding support predicts more episodes of depression and anxiety which could lead to adverse illness outcomes [30]. Stigma contributes to delays in care as women do not want to reveal their condition, and contributes to poor follow up, which potentially leads to ineffective treatment. Stigma and avoidance behavior 
directed towards cancer patients have been reported [31]. Although Joseph et. al., [16] identify patient factors, limited resources, and inadequate medical infrastructure as key contributors to poor follow up, issues around culture and stigma are equally critical. Therefore, psychological interventions for women with breast cancer must always take into account the broader social context in which cancer occurs, with a focus to improving social support.

In conclusion, the quality of breast cancer services in the Mombasa County is complicated by a host of factors including socioeconomic, cultural, health system challenges which influence health outcomes. To improve quality of care for breast cancer, systems for early detection should be put in place including promoting the empowerment of women to access healthcare, developing infrastructure for diagnosis and treatment, and mammography screening.

\section{Competing Interests}

None declared.

\section{Ethical Approval}

Ethical approval was sought from Strathmore University Ethical Review Board (SU-IRB), No. 0085/17.

\section{Ethics, Consent and Permissions}

All participants gave written consent for the study.

\section{Consent for Publication}

All participants consented to the publication of this work.

\section{Availability of Data}

Please contact author for data requests

\section{Funding}

This study had no funding but piggy-backed on ongoing program implementation work.

\section{References}

[1] Novartis Access, Novartis launches affordable treatment options for chronic diseases, in Sunday Nation. 2015, Nation Media Group: Nairobi.

[2] Odeyemi, J., Cancer causes more deaths than HIV/AIDS, tuberculosis, malaria combined- Expert, in Daily Trust. 2016, DailyTrust: Lagos.

[3] Mathers, C.D. and D. Loncar, Projections of global mortality and burden of disease from 2002 to 2030. PLoS Med, 2006. 3(11): p. e442.

[4] The Economist, Cancer in the developing world: Worse than AIDS, in The Economist. 2014, The Economist: United Kingdom.
[5] Mojtehedzadeh, S., Killer cancer still neglected in Kenyan health care system, in Daily Nation. 2013, Nation Media Group: Nairobi.

[6] Kairu, P., The Lord or the doctor, whoever comes first: Probing the state of Kenya's cancer care, in Daily Nation. 2015, Nation Media Group: Nairobi.

[7] Nugent, R., Chronic diseases in developing countries: health and economic burdens. Ann N Y Acad Sci, 2008. 1136: p. 70-9.

[8] Korir, A., et al., Incidence of cancer in Nairobi, Kenya (20042008). Int J Cancer, 2015. 137(9): p. 2053-9.

[9] Ministry of Health, Kenya STEPwise survey for noncommunicable diseases risk factors 2015 report. 2015, Ministry of Health, Department of Non-Communicable Diseases: Nairobi.

[10] Lima, M., F. Kerr-Côrrea, and J. Rehm, Alcohol consumption pattern and Coronary Heart Disease risk in Metropolitan São Paulo: analyses of GENACIS Project. Revista Brasileira de Epidemiologia, 2013. 16(1).

[11] Tawa, N., J. Frantz, and F. Waggie, Risk factors to chronic noncommunicable disease in Mombasa, Kenya: Epidemiological study using WHO step wise approach. African journal of health science, 2011. 19: p. 24-29.

[12] Pan American Health Organization, Alcohol, gender, culture and harms in the Americas: PAHO Multicentric Study final report. 2007, Pan American Health Organization: Washington, DC.

[13] Mwai, D. and M. Muriithi, Non-communicable diseases risk factors and their contribution to NCD incidences in Kenya. European Scientific Journal 2015. 11(30): p. 1857-7881.

[14] Sandelowski, M., Sample size in qualitative research. Res Nurs Health, 1995. 18(2): p. 179-83.

[15] Krejcie, R.V. and D.W. Morgan, Determining sample size for research activities. Educational and Psychological Measurement, 1970. 30: p. 607-610.

[16] Joseph, N., E. Hinchcliff, and A. Goodman, Cervical Cancer Screening: The Challenges of Tracking and Follow-up. Journal of Genital System \& Disorders, 2015. 4.4.

[17] Lindesay, J., et al., Knowledge, uptake and availability of health and social services among Asian Gujarati and white elderly persons. Ethn Health, 1997. 2(1-2): p. 59-69.

[18] Aikins, A., et al., Tackling Africa's chronic disease burden: from the local to the global. Available: https://www.ncbi.nlm.nih.gov/pubmed/20403167, 2010.

[19] Pearson, L. and R. Shoo, Availability and use of emergency obstetric services: Kenya, Rwanda, Southern Sudan, and Uganda. Int J Gynaecol Obstet, 2005. 88(2): p. 208-15.

[20] Vardy, J. and I.F. Tannock, Quality of cancer care. Ann Oncol, 2004. 15(7): p. 1001-6.

[21] Sankaranarayanan, R., et al., Cancer survival in Africa, Asia, and Central America: a population-based study. Lancet Oncol, 2010. 11(2): p. 165-73.

[22] McIntyre, D., et al., What are the economic consequences for households of illness and of paying for health care in low- and middle-income country contexts? Soc Sci Med, 2006. 62(4): p. 858-65.

[23] Russell, S., The economic burden of illness for households in developing countries: a review of studies focusing on malaria, tuberculosis, and human immunodeficiency virus/acquired immunodeficiency syndrome. Am J Trop Med Hyg, 2004. 71(2 Suppl): p. 147-55

[24] Battaglia, T.A., et al., Improving follow-up to abnormal breast cancer screening in an urban population. A patient navigation intervention. Cancer, 2007. 109(2 Suppl): p. 359-67.

[25] Gondos, A., et al., Cancer survival in Kampala, Uganda. Br J Cancer, 2005. 92(9): p. 1808-12.

[26] Mwaniki, M.K., et al., An increase in the burden of neonatal admissions to a rural district hospital in Kenya over 19 years. BMC Public Health, 2010. 10: p. 591.

[27] Chuma, J., V. Okungu, and C. Molyneux, Barriers to prompt and effective malaria treatment among the poorest population in Kenya. Malar J, 2010. 9: p. 144.

[28] Chuma, J., et al., Towards achieving Abuja targets: identifying and addressing barriers to access and use of insecticides treated nets among the poorest populations in Kenya. BMC Public Health, 2010. 10: p. 137.

[29] Mwaka, A.D., et al., Understanding cervical cancer: an exploration of lay perceptions, beliefs and knowledge about cervical cancer among the Acholi in northern Uganda. BMC Womens Health, 2014. 14: p. 84. 
[30] Burgess, C., et al., Depression and anxiety in women with early breast cancer: five year observational cohort study. BMJ, 2005. 330(7493): p. 702.
[31] Chapple, A., S. Ziebland, and A. McPherson, Stigma, shame, and blame experienced by patients with lung cancer: qualitative study. BMJ, 2004. 328(7454): p. 1470. 